This item was submitted to Loughborough's Research Repository by the author.

Items in Figshare are protected by copyright, with all rights reserved, unless otherwise indicated.

\title{
Current advances on deep learning-based human action recognition from videos: a survey
}

PLEASE CITE THE PUBLISHED VERSION

https://doi.org/10.1109/ICMLA52953.2021.00054

PUBLISHER

IEEE

VERSION

AM (Accepted Manuscript)

\section{PUBLISHER STATEMENT}

Personal use of this material is permitted. Permission from IEEE must be obtained for all other uses, in any current or future media, including reprinting/republishing this material for advertising or promotional purposes, creating new collective works, for resale or redistribution to servers or lists, or reuse of any copyrighted component of this work in other works.

\section{LICENCE}

All Rights Reserved

\section{REPOSITORY RECORD}

Zhang, Yixiao, Baihua Li, Hui Fang, and Qinggang Meng. 2022. "Current Advances on Deep Learning-based Human Action Recognition from Videos: A Survey”. Loughborough University. https://hdl.handle.net/2134/16645180.v1. 


\section{Current Advances on Deep Learning-based Human Action Recognition from Videos: a Survey}

\author{
Yixiao Zhang \\ Computer Science \\ Loughborough University \\ Loughborough, UK \\ Y.Zhang8@lboro.ac.uk
}

\author{
Baihua $\mathrm{Li}^{*}$ \\ Computer Science \\ Loughborough University \\ Loughborough, UK \\ B.Li@lboro.ac.uk
}

\author{
Hui Fang \\ Computer Science \\ Loughborough University \\ Loughborough, UK \\ H.Fang@lboro.ac.uk
}

\author{
Qinggang Meng \\ Computer Science \\ Loughborough University \\ Loughborough, UK \\ Q.Meng@lboro.ac.uk
}

\begin{abstract}
Human action recognition (HAR) from RGB videos is essential and challenging in the computer vision field due to its wide range of real-world applications in fields of human behaviour analysis, human-computer interactions, robotics and surveillance etc. Since the breakthrough and fast development of deep learning technology, the performance of HAR based on deep neural networks has been significantly improved in this decade. In this survey, we discuss the growing use of deep learning for HAR, such as representative two-stream and 3D CNNs, and particularly highlight most recent success achieved by using attention and transformers. We will provide our perspective on the new trend of designing innovative deep learning methods. In addition, we also present popular HAR datasets developed in recent years and benchmark accuracy achieved by current advancement in deep learning. This draws research attention to the challenges of HAR by identifying performance gaps when applying the deep learning methods on large HAR datasets. Further, this survey sheds light on the development of new methods and facilitates qualitative comparison with state of the art.

Index Terms-Human action recognition, Deep learning, Convolutional Neural Networks, Transformer.
\end{abstract}

\section{INTRODUCTION}

Video human action recognition (HAR) aims to recognise human actions from videos. It becomes one of the most important tasks in computer vision, driven by a huge range of real-world applications, including human behaviour analysis in surveillance, healthcare, robotics and human-computer interaction, autonomous vehicles, and ambient intelligence [1]-[5].

Video-based HAR has a long history since it was firstly proposed in last century [6]. At its very early stage, features, such as motion energy image [7] and movement trajectory [8], were used for categorising human actions into a handful of predefined classes, with limitation of real-time robust video analysis required. With the improvement of computational power, more advanced feature representations, including space-time shapes-based method [9], space-time neighbourhood featuresbased method [10], and eigenjoints-based method [11] etc., were proposed to further increase the HAR accuracy.

Since convolutional neural network (CNN) was adapted in video-based HAR by [12], deep neural network (DNN) based methods, including two-stream networks, Recurrent Neural Networks (RNNs), 3D CNNs, and most recently attentionbased CNNs and transformers, have been proposed to improve both accuracy and efficiency of human action understanding from videos. Despite significant progress has achieved by using current state of the art, we identify huge performance gaps when applying DNNs to large-scale HAR datasets such as HMDB51 [13] and Charades [14], containing a wide range of indoor and outdoor action classes as norm with daily settings in real-world applications,

Thus, in this paper, a comprehensive survey on video-based HAR is provided to discuss technical details and performance of various types of representative DNNs. We firstly build a roadmap of video-based HAR by analysing the trend of DNNs and discuss their advantage and limitation. This could inspire new HAR algorithm development by understanding the pros and cons of different types of methods. In addition, we introduce the most popular video-based HAR datasets and benchmark performance achieved by representative DNN models. It facilitates objective assessment metrics when new HAR methods are proposed.

The contributions of this paper can be summarised as follows:

- we provide a comprehensive categorical survey on deep learning-based RGB video human action recognition methods with more insight on technical analysis,

- we introduce widely-used large-scale RGB video HAR datasets and benchmark milestone made by DNN based methods for quantitative evaluation and comparison,

- we analyse the advantage and disadvantage of each category of methods to shed light on new algorithms and DNN models development.

The remainder of this survey is organised as follows. In Section II, DNN based methods are discussed and analysed by categorising them into two-stream CNN methods, RNNbased networks, 3D CNN-based approaches, attention-based models, and transformers. Section III introduces RGB video datasets and Section IV benchmarks human action recognition performance of many representative models in these datasets.

\section{RoAdMAP OF DEEP LEARNING-BASED HAR}

We provide a roadmap of DNN based video HAR by using a chronological order as illustrated in Fig. 1. These representative methods are categorised into blocks, including two-stream CNN networks, RNNs, 3D CNNs, attention-based $\mathrm{CNNs}$, and new trend of transformers. 


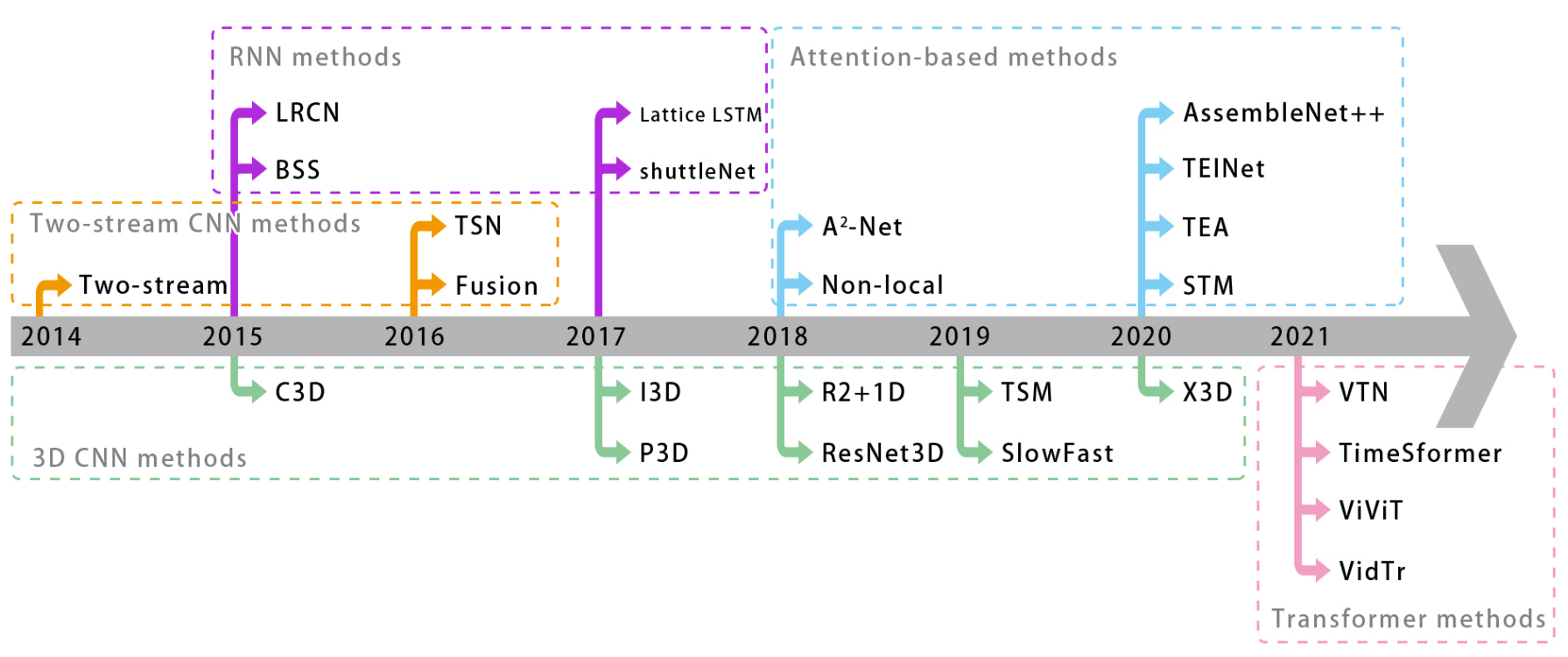

Fig. 1. Some representative methods in video human action recognition by category

\section{A. Two-stream CNN networks}

Two-stream network was originally proposed in [15] for video human action recognition. In this work, two types of feature representations, including spatial feature extracted from individual frames and temporal motion feature extracted from multi-frame dense optical flow fields, are fed into two-stream CNNs for classification. Both streams use VGG backbone [16], which is composed of five 2D convolutional layers and 2 fully connected layers to generate action features, followed by a Softmax layer for each stream. The final classification is built on fusion of the scores from the two-steams. The model was tested on UCF-101 and HMDB-51, demonstrating the potential of optical flow for saving training data. MultiGPU training was introduced to speed up training process. Further, in [17], a new fusion strategy that concatenates these two-stream features at convolutional layers are proposed to achieve a better performance compared to the decision-level fusion used in [15].

To tackle the problem of inability in modelling long-range temporal structure, Temporal Segment Networks (TSN) [18] designs a sparse sampling scheme to represent temporal features. After segmenting a video into $\mathrm{K}$ segments, a sequence of snippets is randomly sampled from these segments to calculate warped optical flow fields. Further, RGB differences and optical flow fields between two consecutive frames are stacked with the warped optical flow fields to improve the efficient and effective learning of original two-stream network.

Two-stream CNN networks are capable of capturing high semantic representations by using $\mathrm{CNNs}$ on both intra/inter-frame spatial-temporal features. However, the precomputing optical flow is computationally expensive and storage-demanding although optical flow is a great temporal representation of human actions.

\section{B. Recurrent Neural Networks}

RNNs have been widely applied in time series analysis due to their capability to model long-term sequential dynamics [19]. As HAR in video requires to learn temporal dynamics from input sequences, RNNs naturally offer solutions to videobased HAR.

Long-term Recurrent Convolutional Networks (LRCN) [20] is one of the first methods using recurrent neural networks to model temporal patterns for human action recognition task. After CNN features are extracted from individual frames, they are fed into LSTM for three different HAR tasks, including activity recognition, image captioning and video description.

Lattice-LSTM [21], or called $L^{2}$ STM, is a two-stream LSTM based method. By adopting a framework used in [15]. LSTMs are integrated into the two streams to further efficiently model the dynamic information in video sequences for better classification.

ShuttleNet [22] is a biologically-inspired deep network which is embedded in a CNN-RNN framework containing several layers of loop-connected GRU processors. Inspired by brain's feedforward and feedback connections, shuttleNet deployed loop connection layers in the network to mimic the brain. Each layer of the network has GRU processors organised as a ring and they are loop connected to the next layer. Attention mechanism is also used to select the best information flow pathway.

\section{C. $3 D$ CNNs}

Recently, 3D CNNs have dominated their HAR performance since they provide end-to-end training architectures [23]-[28]. 3D CNNs implicitly extract temporal features without preprocessing and extracting image or inter-frame motion features from such as optical flows, therefore they significantly reduce computational power and extra feature storage. 
C3D [23] is one of the earliest 3D CNN model for videobased HAR. In this work, it states that $3 \mathrm{D}$ CNNs could be more suitable for temporal modelling compared to $2 \mathrm{D} \mathrm{CNN}$ or using optical flow. The C3D network is simple and efficient as it only contains eight convolution layers, five max pooling layers and two fully connected layers. As there is no need to compute optical flow fields, it is faster than two-stream based methods. To shorten the training process of C3D model, Inflated 3D ConvNet (I3D) [24] was proposed by inflating kernels from a 2D CNN model pretrained on ImageNet. In this manner, the model could achieve better convergence as well as improved training speed.

New deep network backbone architectures, e.g. ResNeXt [29] and EfficientNet [30], have shown their superiority when being adapted to 3D CNNs. In [25], ResNet3D based on ResNeXt-101 network is trained to achieve the state-of-the-art performance on the Kinetics dataset [31]. X3D network [28] extends EfficientNet by increasing the number of expansion axes to six axes which include temporal duration, frame rate, spatial resolution, network width, bottleneck width, and depth. It also demonstrates competitive performance with much fewer parameters and simpler network architecture compared to classical 3D CNN architectures.

Mult-scale strategies have also been considered in temporal dimensions of 3D CNNs. For example, in [27], SlowFast network contains two 3D CNN networks to process a slow pathway and a fast pathway which operate at different frame rate. These two pathways potentially combine temporal patterns from both holistic and granular levels to understand human actions in video sequences. Similarly, coarse-fine network [32] is another two pathway method. It contains a coarse stream and a fine stream that process most informative key frames and all frames respectively. There is a Grid Pool module at the head of the coarse stream which could select the most crucial frames among the full video using a cumulative distribution based downsampling algorithm.

Building fast lightweight 3D CNN architecture is another research focus to solve the major bottleneck of $3 \mathrm{D} \mathrm{CNN}$ for HAR to reduce computational cost and high consumption of memory. In [33], a pseudo 3D (P3D) residual network was proposed to reduce both computation and memory demand. Due to the success of $3 \times 3 \times 3$ convolution in C3D, P3D network uses two CNNs, $1 \times 3 \times 3$ convolution on spatial domain and $3 \times 1 \times 1$ convolution on temporal domain, to simulate one 3D CNN in C3D. When applying P3D block to ResNets, it only increases model size by less than $6 \%$ but improves the overall performance by $3.4 \%$. R $(2+1) \mathrm{D}$ network [26] also adapted the idea of using simplified CNN to substitute $3 \mathrm{D} \mathrm{CNN}$ to reduce convolution computation in 3D space. Different from P3D, R(2+1)D network abandons bottleneck structure and only uses a single type of spatialtemporal residual block in all layers. Another difference is that $\mathrm{R}(2+1) \mathrm{D}$ uses $1 \times d \times d$ convolution on spatial domain and $t \times 1 \times 1$ convolution on temporal domain to simulate $t \times d \times d$ 3D convolution which makes $2 \mathrm{D}$ convolutions have comparable parameters with the 3D convolutions. In [34],
Temporal Shift Module (TSM) network provides an alternative way to simplify 3D CNN architecture by shifting temporal dimension of neighbouring frames before convolving with the input tensor.

\section{Attention-based Models and Transformers}

Attention and transformer mechanism are emerging to enhance feature representation via matching queries with key salient features, so that long range dependency on both spatial and temporal space could be captured effectively. Different from CNN that can only process one local neighbourhood at a time, the attention mechanism allows networks to gain information in certain distance which significantly enhance both temporal and spatial modelling performance. It has been evidenced significant increase of using attention-based models and transformers in HAR [35]-[41].

Attentional Pooling [35] is one of the first methods that introduces attention mechanism in video-based HAR. The attentional pooling is an efficient low-rank approximation to second order pooling. The attentional pooling is described as a module or a layer that replaces a pooling layer in a standard CNN to remain crucial features based on attention weights. The result shows that attentional pooling provides solid improvement over baselines.

Non-local network [36] was designed to capture long-range dependencies using non-local blocks. The spacetime nonlocal block in non-local network performs like a self-attention module which consists of several convolutions, computing the local response as a weighted sum of features. Non-local block has the same dimension of input and output, so it could be inserted into many existing networks to improve the spacetime modelling performance of the network. The non-local block is a simple block that only increases a little model size, but could give a significant accuracy improvement on existing networks.

Double Attention Networks ( $\mathrm{A}^{2}$-Nets) [37] contains double attention blocks which use two attention maps to gather and distribute long-range features. The first attention module, which is applied to the entire spatial space, generates global features. The second attention module using attention pooling similar to [35] to adaptively extract local features at each spatio-temporal location. The double attention block also keeps the size of the tensor stable and could be inserted into many existing networks.

AssembleNet++ [42] introduces peer-attention which is a form of channel-wise attention. Peer-attention could learn the attention weights dynamically in the network. In other words, it could learn the importance of spatio-temporal features at each convolutional block. Architecture search for neural attention connectivity is also explored in AssembleNet++ to gain better connectivity.

SpatioTemporal and Motion (STM) network [43] models both spatial and temporal characteristics in a unified CNN framework. STM network is based on ResNet architecture and replaces all residual blocks using STM blocks. An STM block includes two parallel modules, which are Channel-wise 
SpatioTemporal Module (CSTM) and Channel-wise Motion Module (CMM). The CSTM is designed to extract spatial features and coarse temporal features whilst CMM is used to capture short motion feature between adjacent frames.

TEINet [38] is also based on ResNet architecture. Compared to the STM network [43], Temporal Enhancement AndInteraction (TEI) block keeps the shape of the input tensor and could be directly inserted into residual blocks in ResNet rather than replacinge residual blocks. A TEI block also contains two modules connected in series, a Motion Enhanced Module (MEM) followed by a Temporal Interaction Module (TIM). MEM is a temporal attention model that designed to enhance the performance of short motion modelling by channel-wise convolutions, while TIM is proposed to enhance temporal contextual information extraction of the ResNet. Very similar to TEINet, TEA network [44] has Temporal Excitation and Aggregation (TEA) block that can be inserted into ResNet and contains a motion excitation (ME) module and a multiple temporal aggregation (MTA) module. ME module works similar to MEM which captures feature-level temporal differences between adjacent frames. MTA module is designed to extract long-range temporal feature as TIM.

Recently, transformers, as an emerging model to capture long-term temporal dependencies, has become to draw increasing attention for video-based HAR. In [45], a Video Action Transformer Network was designed to recognise and localise human and their actions in video clips. In this work, I3D network [24] is used to extract convolutional feature maps to feed into a region proposal network. It is followed by a transformer architecture which is consist of action transformer units. Action transformer units aim to enhance the feature between people and objects. The result indicates that the Transformer could work well with convolutions.

Video Transformer Network (VTN) [46] was proposed in 2021. VTN uses a ViT [39] backbone for spatial modelling and an attention-based encoder for temporal modelling. ViT [39] is another Transformer for image classification which is used as a 2D spatial feature extraction module. The sequence of feature extracted from frames are then input to the attentionbased encoder as tokens. An MLP module is used at the end of the network for class prediction. The experiments show that a simple combination of spatial transformer and temporal transformer could give a comparable result with I3D [24] on the video human action recognition task.

TimeSformer [41] discussed further about how to combine spatial transformer and temporal transformer. Four types of combination structures with different complexity are explored, namely Joint Space-Time Attention, Divided Space-Time Attention, Sparse Local Global Attention, and Axial Attention. Joint Space-Time Attention is a simple model that uses single attention to model both spatial and temporal information. Sparse Local Global Attention firstly uses neighbouring patches to get local attention and then compute global attention in both spatial and temporal dimension from the entire clip. Axial Attention uses three attention modules for three dimensions of time, width of the frame and height of the frame

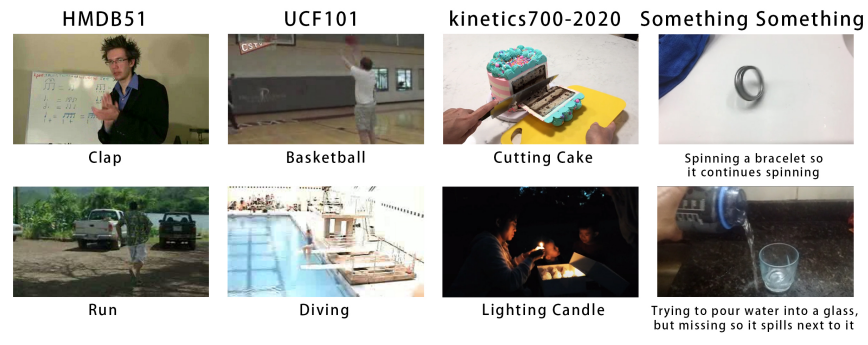

Fig. 2. Samples from HMDB51 [13], UCF101 [48], Kinetics 700-2020 [49], and Something Something [50] dataset

respectively. Divided Space-Time Attention is finally adapted for TimeSformer which apply temporal attention first followed by spatial attention. The research shows that Divided SpaceTime Attention structure has advantages in both efficiency and accuracy as Table II shows.

ViViT [47] is another transformer for video human action recognition just released in 2021. The paper introduced four types of structures for spatial and temporal transformer combination which have nearly same complexity, namely spatio-temporal attention, Factorised encoder, Factorised self-attention, and Factorised dot-product attention. Spatio-temporal attention is similar to Joint Space-Time Attention in TimeSformer [41], while Factorised encoder is similar to VTN [46]. Factorised self-attention has the same number of transformer layers with spatio-temporal attention but computes self-attention on both spatial and temporal dimensions separately. Factorised dot-product attention uses multi-head dotproduct attention instead of multi-headed self-attention. Multihead dot-product attention has spatial heads and temporal heads which only compute spatial dimensions and temporal dimension in the corresponding head. Spatio-temporal attention is the current state-of-art method on Kinetics 600 dataset.

\section{DATASET}

During this decade, many large-scale HAR dataset, e.g., HMDB51, UCF101, Kinetics, Something Something etc., have been released in order to evaluate and compare different approaches. Samples in these datasets are illustrated in Fig. 2. In this section, we introduce 14 popular HAR datasets which have been widely used for performance comparison of DNN based models.

HMDB51 dataset [13] was introduced in 2011. It is one of the earliest large-scale datasets that introduced to evaluate human action recognition approaches. 7,000 short video clips from a variety of sources ranging from digitized movies to YouTube were manually extracted and annotated. A total of 51 daily action classes was collected from indoor and outdoor. The actions in this dataset are atomic, for example, catch, jump, punch.

UCF50 dataset [51] was released in 2011. UCF50 dataset contains 50 action classes with a total of 6676 video samples collected from YouTube. Each class has more than 100 video samples. The actions list contains sports and a small session of 


\begin{tabular}{|l|l|l|l|l|l|l|}
\hline Dataset Name & Classes & Samples & Length & Video Source & Content & Year \\
\hline \hline HMDB51 [13] & 51 & $7 \mathrm{~K}$ & $\sim 5 \mathrm{~s}$ & YouTube/Movies & Action & 2011 \\
UCF50 [51] & 50 & $7 \mathrm{~K}$ & $\sim 6 \mathrm{~s}$ & YouTube & Sports & 2012 \\
UCF101 [48] & 101 & $13 \mathrm{~K}$ & $\sim 7 \mathrm{~s}$ & YouTube & Sports & 2012 \\
Kinetics 400 [31] & 400 & $306 \mathrm{~K}$ & $\sim 10 \mathrm{~s}$ & YouTube & Human activities & 2017 \\
Kinetics 600 [52] & 600 & $496 \mathrm{~K}$ & $\sim 10 \mathrm{~s}$ & YouTube & Human activities & 2018 \\
Kinetics 700 [53] & 700 & $650 \mathrm{~K}$ & $\sim 10 \mathrm{~s}$ & YouTube & Human activities & 2019 \\
Kinetics 700-2020 [49] & 700 & $648 \mathrm{k}$ & $\sim 10 \mathrm{~s}$ & YouTube & Human activities & 2020 \\
Sth-Sth-v1 [50] & 174 & $109 \mathrm{~K}$ & $\sim 4 \mathrm{~s}$ & YouTube/ Google Image & Action & 2017 \\
Sth-Sth-v2 [54] & 174 & $221 \mathrm{~K}$ & $\sim 4 \mathrm{~s}$ & YouTube/ Google Image & Action & 2018 \\
Sports1M [12] & 487 & $1.1 \mathrm{M}$ & $\sim 5 \mathrm{~s}$ & YouTube & Sports & 2014 \\
Activitynet [55] & 200 & $24 \mathrm{~K}$ & $\sim 5 \mathrm{~m}$ & YouTube & Human activities & 2015 \\
Youtube-8m [56] & 3862 & $8.2 \mathrm{M}$ & $\sim 4 \mathrm{~m}$ & YouTube & Indoor activities & 2016 \\
Charades [14] & 157 & $10 \mathrm{~K}$ & $\sim 30 \mathrm{~s}$ & Newly captured & Human activities & 2020 \\
HVU [57] & 739 & $572 \mathrm{~K}$ & $\sim 10 \mathrm{~s}$ & YouTube & & \\
\hline
\end{tabular}

TABLE I

A LIST OF POPULAR RGB DATASETS OF VIDEO HUMAN ACTION RECOGNITION

music instrument playing. UCF101 dataset [48] was released in 2012. It is an extension of UCF50 including 50 action classes in UCF50 dataset and 51 new classes. The size of UCF101 dataset doubled by UCF50 dataset to $13 \mathrm{~K}$ video samples. Most of the action classes in UCF101 are sports, like basketball, high jump, surfing, skiing. UCF101 is one of the most commonly used dataset due to the amount of action classes presented and variation of each class in various indoor/outdoor settings required for DNN training.

With the revolution of deep learning e.g. transformers, the HMDB51 and UCF101 limit themselves on providing enough training data, especially they do not have sufficient variation of an action class. Thanks to Kinetics datasets [31], [49], [52], [53] which is updated annually in the past few years. In 2017, Kinetics-400 dataset [31] was developed to provide a new challenge. Kinetics-400 [31] contains 400 activity classes and more than 300,000 10-second video clips which are extracted from YouTube. In 2018, Kinetics-600 [52] was introduced and 100 more classes are added. Kinetics-700 [53] was released in the following year with additional 100 classes. However, Kinetics datasets only provided YouTube links rather than video clips which means the dataset cloud change and reduce when online videos are removed or set to private. To deal with this problem, Kinetics-700-2020 [49] is currently annually updated based replacing removed videos with new ones. Nowadays Kinetics dataset is the most popular dataset due to its large size and a wide range of video resource from the world-wide media platform YouTube. It provides the potential of sufficient training data for state-of-the-art deep learning models including the most data demanding transformer networks.

Something-Something dataset [50], [54] is a strong temporal-related dataset. Unlike other datasets focusing on human activities, action classes in this dataset present human interaction with objects, for example, "moving something up" and "pushing something from left to right". One feature of this dataset is that it does not specify what object is interacting with people and it just uses something instead. Both the v1 [50] and v2 [54] versions contain 174 classes. The later version has a double number of videos than version 1 which has about
$221 \mathrm{~K}$ videos. Each video in the dataset is about $2 \sim 6$ seconds. This dataset is often used to evaluate temporal evolution for human-object interaction.

Sports-1M dataset [12] consists of one million videos annotated with 487 classes. Each class contains about 1000-3000 video clips. Videos in Sports-1M are collected automatically by YouTube Topics API. The quality of the data may vary from manually annotated data, and using this dataset needs to consider facing the problem of the number of videos available decreasing.

Activitynet dataset [55] was introduced in 2015. It is a human activities dataset that contains 200 activity classes and about $24 \mathrm{~K}$ videos. The length of video varies from a few seconds to over ten minutes.

Youtube-8M dataset [56] was released by Google Research in 2016. As its name, the Youtube-8M dataset composed of about 8 million videos. There are 3862 classes covering sports, daily activities in this dataset, and each video could have several different classes, for example 3 action classes with length of 230 seconds on average.

Charades dataset [14] was introduced in 2016. This dataset is composed of $9.8 \mathrm{~K}$ videos with an average length of 30 seconds. The dataset was collected by crowdsourcing workers to capture videos according to the scripts they provided in their home. All activities in this dataset are indoor activities, such as "watching TV", "sneezing" which provides a dataset for homecare and indoor surveillance related applications.

HVU dataset (Holistic Video Understanding) [57] was recently released in 2020. HVU dataset sets up three tasks including video classification, video captioning, and video clustering. The data in this dataset is collected from YouTube$8 \mathrm{M}$ [56], Kinetics-600 [52] and HACS [58]. The class label is automatically generated by Google Vision API and validated by human. The dataset contains 739 classes and 572K videos. Each video is around 10 seconds.

Table I lists these popular datasets. Among them, HMDB51 and UFC101 are commonly used for baseline comparison. From 2017, Kinetics dataset family became increasingly popular dataset as 3D convolutional approaches can only achieve their perform benefit when training on a much large dataset. 
Kinetics provides nearly a hundred times the amount of data compared to HMDB51 and therefore is wildly used for pretraining a model. Kinetics dataset is now used as the baseline in video human action recognition. Something-Something dataset is also wildly used in the past three years, as it is a strong temporal-related dataset. It has a desirable size that is not too large to cause time difficulty in training and not too small to lead to convergence difficulty. Table I also summarises several other popular datasets at the end. As Table I shows, some datasets were developed for some specific domain, which may be used for some specific application, e.g. indoor surveillance. Some datasets often used for pre-train a model which could lead a better performance in the baseline, e.g. Sports-1M.

\section{Discussion}

Different methods for video HAR are classified into four categories as in the previous section. Two-stream methods and RNN methods were mainstream methods before 2017, while 3D CNN methods became popular later. Recently, transformer-based methods have become a new trend. In this section, the performance of these representative models on widely-used benchmark datasets are presented firstly. Then, the difficulty of model training and the importance of pretraining are discussed. Finally, model capacity and deploy ability are discussed.

Accuracy: Table II shows the performance of representative methods on five datasets. Two-stream CNNs methods and RNNs methods were first introduced and evaluated on UCF101 dataset and HMDB51 dataset. Thanks to the improvement of computational power in recent years, a GPU could accommodate larger networks which enable training of a larger network. 3D CNNs methods exceeded performance of twostream methods and RNNs methods universally on accuracy as Table II shows. As many methods have achieved more than 97\% accuracy on UCF101, Kinetics dataset are introduced to evaluate accuracy for $3 \mathrm{D}$ CNNs methods. Due to the drawback of high computation cost and high consumption of memory for 3D CNNs, some simplified CNNs methods are introduced to model spatial information and temporal information separately and increased the accuracy to a new stage. Recently, transformer-based methods represent state-ofart performance in video human action recognition. Comparing with 3D CNNs, self-attention also has advantage to capture both short motion and long range dependencies.

Training: In the view of pre-training, due to the huge number of parameters, pre-training is very important to train a deep and large model. UCF101 and HMDB51 possess only several thousands of samples for each class which not be enough to train a very deep 2D/3D CNN or transformer from scratch. To reduce required training data, most models listed in Table II are pre-trained using e.g. Kinetics400. To further reduce training cost, many methods also adapt pre-trained weight directly from image classification models. For example, I3D inflates both network structure and network weights from a 2D CNN model pretrained on ImageNet. Transformer-based methods have much more trainable parameter than 3D CNN which normally requires a pre-training process on the images, e.g. TimeSformer is built on a pre-trained ViT that trained on ImageNet.

In the view of difficulty in training, two-stream CNNs using 2D convolution kennels are relatively easy to train comparing to 3D CNNs and transformers with large network parameters. RNNs also benefit in training due to their simple structure and smaller set of model parameters to reduce underfitting risk when lack of training samples. However, two-stream CNNs and RNN-based model normally request optical flow as one stream of input, pre-computing optical flow not only costs a huge amount of computational power, but also a significant storage-demand. 3D CNNs are hard to train as their huge number of trainable parameters. Comparing with 3D CNNs, transformer-based methods normally have lower FLOPs (floating point operations) which is fast to train but it needs larger dataset than 3D CNNs.

Capacity and deployability: The two-stream CNNs methods and RNNs models have a small model capacity of parameters, but difficulty in pre-computing optical flow makes them hard to deploy. 3D CNNs has a medium number of parameters which is much larger than $2 \mathrm{D}$ CNNs and smaller than transformer-based models. Larger models with a high number of parameters could lead to high computation cost in FLOPs. Transformers have the most trainable parameters by using multi-heads attention. However, due to the different between convolution and self- attention, transformers normally have much fewer FLOPs than 3D CNNs although it need more memory, for example, TimeSformer [41] has about 4 times of parameters than I3D network [24], but only needs about $1 / 3$ inference time of the I3D. Therefore, transformers have better real-time inference ability when larger memory available.

\section{CONCLUSION}

Over the past decade, deep learning-based video HAR methods have made remarkable achievement. This paper provides a survey of $2 \mathrm{D} \mathrm{CNN}, \mathrm{RNN}, 3 \mathrm{D} \mathrm{CNN}$, attention-based and transformer approaches to deep learning-based HAR. Although this paper maybe not an exhaustive list, we hope that this paper could give the representative approaches in each category in recent years. A comprehensive description of widely used datasets in HAR area are summarised.

Although the achievements in this area have recently paid off, the research of HAR still needs further study. A clear trend can be observed that transformer-based methods are becoming popular methods compared to modern 3D CNNs to solving HAR which have great accuracy, low training cost, low inference cost, and good capacity. In the field of HAR, a larger dataset is also needed to meet the demand of transformers training.

\section{ACKNOWLEDGMENT}

The first author would like to thank Chinese Scholarship Council (CSC) and Loughborough University for supporting his study. 


\begin{tabular}{|c|c|c|c|c|c|c|c|}
\hline Name & Venue & UCF101 & HMDB51 & Kinetics 400 & Kinetics600 & Charades & Pre-training \\
\hline $\begin{array}{l}\text { Two-stream CNNs methods } \\
\text { Two-stream [15] } \\
\text { Two-Stream Fusion [17] } \\
\text { TSN [18] }\end{array}$ & $\begin{array}{c}\text { NIPS } 14 \\
\text { CVPR } 16 \\
\text { ECCV } 16\end{array}$ & $\begin{array}{l}88.0 \\
92.5 \\
94.2 \\
\end{array}$ & $\begin{array}{l}59.4 \\
65.4 \\
69.4 \\
\end{array}$ & & & & $\begin{array}{l}\text { I } \\
\text { I } \\
\text { I }\end{array}$ \\
\hline $\begin{array}{l}\text { RNNs methods } \\
\text { LRCN [20] } \\
\text { Beyond Short Snippets [59] } \\
\text { Lattice LSTM [21] } \\
\text { shuttleNet [22] } \\
\end{array}$ & $\begin{array}{l}\text { CVPR } 15 \\
\text { CVPR } 15 \\
\text { ICCV } 17 \\
\text { ICCV } 17 \\
\end{array}$ & $\begin{array}{l}82.7 \\
88.6 \\
93.6 \\
95.4 \\
\end{array}$ & $\begin{array}{l}66.2 \\
71.7 \\
\end{array}$ & & & & $\begin{array}{l}\text { I } \\
\text { I } \\
\text { I } \\
\text { I }\end{array}$ \\
\hline $\begin{array}{l}\text { 3D CNNs methods } \\
\text { C3D [23] } \\
\text { RGB-I3D [24] } \\
\text { Two-Stream I3D [24] } \\
\text { ResNet3D (ResNeXt-101) [25] } \\
\text { R2+1D [26] } \\
\text { TSM [34] } \\
\text { SlowFast [27] } \\
\text { X3D-XXL [28] }\end{array}$ & $\begin{array}{l}\text { ICCV } 15 \\
\text { CVPR } 17 \\
\text { CVPR } 17 \\
\text { CVPR } 18 \\
\text { CVPR } 18 \\
\text { ICCV } 19 \\
\text { ICCV } 19 \\
\text { CVPR } 20\end{array}$ & $\begin{array}{l}82.3 \\
95.4 \\
\mathbf{9 7 . 9} \\
94.5 \\
\mathbf{9 7 . 3} \\
95.9\end{array}$ & $\begin{array}{c}56.8[60] \\
74.5 \\
\mathbf{8 0 . 2} \\
70.2 \\
\mathbf{7 8 . 7} \\
73.5\end{array}$ & $\begin{array}{c}59.5[60] \\
72.1[36] \\
75.7[36] \\
65.1[60] \\
75.4 \\
74.7[41] \\
79.8 \\
80.4\end{array}$ & 81.8 & 45.2 & $\begin{array}{l}\text { S } \\
\text { I, K } \\
\text { I, K } \\
\text { S, K }\end{array}$ \\
\hline $\begin{array}{l}\text { Attention-based methods } \\
\mathrm{A}^{2} \text {-Net [37] } \\
\text { Non-local I3D [36] } \\
\text { AssembleNet++ [61] } \\
\text { STM [43] } \\
\text { TEINet [38] } \\
\text { TEA [44] }\end{array}$ & $\begin{array}{l}\text { NIPS } 18 \\
\text { CVPR } 18 \\
\text { ECCV } 20 \\
\text { ICCV } 20 \\
\text { AAAI } 20 \\
\text { CVPR } 20 \\
\end{array}$ & $\begin{array}{l}96.2 \\
96.7 \\
96.9\end{array}$ & $\begin{array}{l}72.2 \\
72.1 \\
73.3\end{array}$ & $\begin{array}{l}74.6 \\
77.7 \\
\\
73.7 \\
76.2 \\
76.1 \\
\end{array}$ & & 59.8 & $\begin{array}{c}\mathrm{K} \\
\mathrm{K} \\
\mathrm{K} \\
\mathrm{I}, \mathrm{K} \\
\mathrm{K}\end{array}$ \\
\hline $\begin{array}{l}\text { Transformer methods } \\
\text { VTN [46] } \\
\text { TimeSformer-L [41] } \\
\text { ViViT-H [47] } \\
\text { VidTr-L [62] }\end{array}$ & $\begin{array}{l}\operatorname{arXiv} 21.02 \\
\operatorname{arXiv} 21.02 \\
\text { arXiv } 21.03 \\
\text { arXiv } 21.04\end{array}$ & 96.7 & 74.4 & $\begin{array}{l}78.6 \\
\mathbf{8 0 . 7} \\
\mathbf{8 4 . 8} \\
79.1\end{array}$ & $\begin{array}{l}85.2 \\
85.8\end{array}$ & 43.5 & $\begin{array}{l}\mathrm{I} \\
\mathrm{I} \\
\mathrm{J} \\
\mathrm{K}\end{array}$ \\
\hline
\end{tabular}

TABLE II

COMPARISON OF DIFFERENT METHODS. ARXIV 21.02 REFERS TO PUbLISH ON ARXIV IN FebruARY 2021. PRE-TRAINING DATASETS: IMAGENET (I) [63], Sports 1M (S) [12], Kinetics (K) [31], JFT (J) [64]. The EVAluation METRICS ARE TOP-1 ACCURACY For UCF101, HMDB51, KINETICS400, KINETICS600 AND MEAN AVERAGE PRECISION (MAP) FOR CHARADES.

\section{REFERENCES}

[1] J. Choi, Y.-i. Cho, T. Han, and H. S. Yang, "A view-based real-time human action recognition system as an interface for human computer interaction," in International Conference on Virtual Systems and Multimedia. Springer, 2007, pp. 112-120.

[2] A. A. Chaaraoui, P. Climent-Pérez, and F. Flórez-Revuelta, "A review on vision techniques applied to human behaviour analysis for ambientassisted living," Expert Systems with Applications, vol. 39, no. 12, pp. 10873-10888, 2012.

[3] M. A. Khan, K. Javed, S. A. Khan, T. Saba, U. Habib, J. A. Khan, and A. A. Abbasi, "Human action recognition using fusion of multiview and deep features: an application to video surveillance," Multimedia tools and applications, pp. 1-27, 2020.

[4] H. Meng, N. Pears, and C. Bailey, "A human action recognition system for embedded computer vision application," in 2007 IEEE Conference on Computer Vision and Pattern Recognition. IEEE, 2007, pp. 1-6.

[5] R. Poppe, "A survey on vision-based human action recognition," Image and vision computing, vol. 28, no. 6, pp. 976-990, 2010.

[6] X. Zhen and L. Shao, "Introduction to human action recognition," Wiley Encyclopedia of Electrical and Electronics Engineering, pp. 1-11, 1999.

[7] A. Bobick and J. Davis, "An appearance-based representation of action," in Proceedings of 13th International Conference on Pattern Recognition, vol. 1. IEEE, 1996, pp. 307-312.

[8] R. Rosales and S. Sclaroff, "Trajectory guided tracking and recognition of actions," PAMI Special Issue on Video Surveillance and Monitoring, 1999.

[9] M. Blank, L. Gorelick, E. Shechtman, M. Irani, and R. Basri, "Actions as space-time shapes," in Tenth IEEE International Conference on Computer Vision (ICCV'05) Volume 1, vol. 2. IEEE, 2005, pp. 13951402.

[10] A. Kovashka and K. Grauman, "Learning a hierarchy of discriminative space-time neighborhood features for human action recognition," in
2010 IEEE computer society conference on computer vision and pattern recognition. IEEE, 2010, pp. 2046-2053.

[11] X. Yang and Y. L. Tian, "Eigenjoints-based action recognition using naive-bayes-nearest-neighbor," in 2012 IEEE computer society conference on computer vision and pattern recognition workshops. IEEE, 2012, pp. 14-19.

[12] A. Karpathy, G. Toderici, S. Shetty, T. Leung, R. Sukthankar, and L. Fei-Fei, "Large-scale video classification with convolutional neural networks," in CVPR, 2014.

[13] H. Kuehne, H. Jhuang, E. Garrote, T. Poggio, and T. Serre, "Hmdb: a large video database for human motion recognition," in 2011 International conference on computer vision. IEEE, 2011, pp. 2556-2563.

[14] G. A. Sigurdsson, G. Varol, X. Wang, A. Farhadi, I. Laptev, and A. Gupta, "Hollywood in homes: Crowdsourcing data collection for activity understanding," 2016.

[15] K. Simonyan and A. Zisserman, "Two-stream convolutional networks for action recognition in videos," Advances in Neural Information Processing Systems (NeurIPS), 2014.

[16] — "Very deep convolutional networks for large-scale image recognition," arXiv preprint arXiv:1409.1556, 2014.

[17] C. Feichtenhofer, A. Pinz, and A. Zisserman, "Convolutional two-stream network fusion for video action recognition," in Proceedings of the IEEE conference on computer vision and pattern recognition, 2016, pp. 19331941.

[18] L. Wang, Y. Xiong, Z. Wang, Y. Qiao, D. Lin, X. Tang, and L. Van Gool, "Temporal segment networks: Towards good practices for deep action recognition," in European conference on computer vision. Springer, 2016, pp. 20-36.

[19] S. Hochreiter and J. Schmidhuber, "Long short-term memory," Neural computation, vol. 9, no. 8, pp. 1735-1780, 1997.

[20] J. Donahue, L. Anne Hendricks, S. Guadarrama, M. Rohrbach, S. Venugopalan, K. Saenko, and T. Darrell, "Long-term recurrent convolutional networks for visual recognition and description," in Proceedings of the 
IEEE conference on computer vision and pattern recognition, 2015, pp. 2625-2634.

[21] L. Sun, K. Jia, K. Chen, D.-Y. Yeung, B. E. Shi, and S. Savarese, "Lattice long short-term memory for human action recognition," in Proceedings of the IEEE international conference on computer vision, 2017, pp. $2147-2156$.

[22] Y. Shi, Y. Tian, Y. Wang, W. Zeng, and T. Huang, "Learning longterm dependencies for action recognition with a biologically-inspired deep network," in Proceedings of the IEEE International Conference on Computer Vision, 2017, pp. 716-725.

[23] D. Tran, L. Bourdev, R. Fergus, L. Torresani, and M. Paluri, "Learning spatiotemporal features with 3d convolutional networks," in Proceedings of the IEEE international conference on computer vision, 2015, pp. $4489-4497$.

[24] J. Carreira and A. Zisserman, "Quo vadis, action recognition? a new model and the kinetics dataset," in proceedings of the IEEE Conference on Computer Vision and Pattern Recognition, 2017, pp. 6299-6308.

[25] K. Hara, H. Kataoka, and Y. Satoh, "Can spatiotemporal 3d cnns retrace the history of $2 \mathrm{~d}$ cnns and imagenet?" in Proceedings of the IEEE conference on Computer Vision and Pattern Recognition, 2018, pp. $6546-6555$.

[26] D. Tran, H. Wang, L. Torresani, J. Ray, Y. LeCun, and M. Paluri, "A closer look at spatiotemporal convolutions for action recognition," in Proceedings of the IEEE conference on Computer Vision and Pattern Recognition, 2018, pp. 6450-6459.

[27] C. Feichtenhofer, H. Fan, J. Malik, and K. He, "Slowfast networks for video recognition," in Proceedings of the IEEE/CVF International Conference on Computer Vision, 2019, pp. 6202-6211.

[28] C. Feichtenhofer, "X3d: Expanding architectures for efficient video recognition," in Proceedings of the IEEE/CVF Conference on Computer Vision and Pattern Recognition, 2020, pp. 203-213.

[29] S. Xie, R. Girshick, P. Dollár, Z. Tu, and K. He, "Aggregated residual transformations for deep neural networks," in Proceedings of the IEEE conference on computer vision and pattern recognition, 2017, pp. 1492 1500 .

[30] M. Tan and Q. Le, "Efficientnet: Rethinking model scaling for convolutional neural networks," in International Conference on Machine Learning. PMLR, 2019, pp. 6105-6114.

[31] W. Kay, J. Carreira, K. Simonyan, B. Zhang, C. Hillier, S. Vijayanarasimhan, F. Viola, T. Green, T. Back, P. Natsev, M. Suleyman, and A. Zisserman, "The kinetics human action video dataset," 2017.

[32] K. Kahatapitiya and M. S. Ryoo, "Coarse-fine networks for temporal activity detection in videos," in Proceedings of the IEEE/CVF Conference on Computer Vision and Pattern Recognition, 2021.

[33] Z. Qiu, T. Yao, and T. Mei, "Learning spatio-temporal representation with pseudo-3d residual networks," in proceedings of the IEEE International Conference on Computer Vision, 2017, pp. 5533-5541.

[34] J. Lin, C. Gan, and S. Han, "Tsm: Temporal shift module for efficient video understanding," in Proceedings of the IEEE/CVF International Conference on Computer Vision, 2019, pp. 7083-7093.

[35] R. Girdhar and D. Ramanan, "Attentional pooling for action recognition," arXiv preprint arXiv:1711.01467, 2017.

[36] X. Wang, R. Girshick, A. Gupta, and K. He, "Non-local neural networks," in Proceedings of the IEEE conference on computer vision and pattern recognition, 2018, pp. 7794-7803.

[37] Y. Chen, Y. Kalantidis, J. Li, S. Yan, and J. Feng, "A ${ }^{2}$-nets: Double attention networks," arXiv preprint arXiv:1810.11579, 2018.

[38] Z. Liu, D. Luo, Y. Wang, L. Wang, Y. Tai, C. Wang, J. Li, F. Huang, and T. Lu, "Teinet: Towards an efficient architecture for video recognition," in Proceedings of the AAAI Conference on Artificial Intelligence, vol. 34 , 2020, pp. $11669-11676$.

[39] A. Dosovitskiy, L. Beyer, A. Kolesnikov, D. Weissenborn, X. Zhai, T. Unterthiner, M. Dehghani, M. Minderer, G. Heigold, S. Gelly, J. Uszkoreit, and N. Houlsby, "An image is worth 16x16 words: Transformers for image recognition at scale," in International Conference on Learning Representations, 2021. [Online]. Available: https://openreview.net/forum?id=YicbFdNTTy

[40] N. Carion, F. Massa, G. Synnaeve, N. Usunier, A. Kirillov, and S. Zagoruyko, "End-to-end object detection with transformers," in European Conference on Computer Vision. Springer, 2020, pp. 213 229.

[41] G. Bertasius, H. Wang, and L. Torresani, "Is space-time attention all you need for video understanding?" arXiv preprint arXiv:2102.05095, 2021.
[42] M. S. Ryoo, A. J. Piergiovanni, J. Kangaspunta, and A. Angelova, "Assemblenet++: Assembling modality representations via attention connections," in Computer Vision - ECCV 2020, A. Vedaldi, H. Bischof, T. Brox, and J.-M. Frahm, Eds. Cham: Springer International Publishing, 2020, pp. 654-671.

[43] B. Jiang, M. Wang, W. Gan, W. Wu, and J. Yan, "Stm: Spatiotemporal and motion encoding for action recognition," in Proceedings of the IEEE/CVF International Conference on Computer Vision, 2019, pp. 2000-2009.

[44] Y. Li, B. Ji, X. Shi, J. Zhang, B. Kang, and L. Wang, "Tea: Temporal excitation and aggregation for action recognition," in Proceedings of the IEEE/CVF Conference on Computer Vision and Pattern Recognition, 2020, pp. 909-918.

[45] R. Girdhar, J. Carreira, C. Doersch, and A. Zisserman, "Video action transformer network," in Proceedings of the IEEE/CVF Conference on Computer Vision and Pattern Recognition, 2019, pp. 244-253.

[46] D. Neimark, O. Bar, M. Zohar, and D. Asselmann, "Video transformer network," arXiv preprint arXiv:2102.00719, 2021.

[47] A. Arnab, M. Dehghani, G. Heigold, C. Sun, M. Lučić, and C. Schmid, "Vivit: A video vision transformer," arXiv preprint arXiv:2103.15691, 2021.

[48] K. Soomro, A. R. Zamir, and M. Shah, "Ucf101: A dataset of 101 human actions classes from videos in the wild," arXiv preprint arXiv:1212.0402, 2012.

[49] L. Smaira, J. Carreira, E. Noland, E. Clancy, A. Wu, and A. Zisserman, "A short note on the kinetics-700-2020 human action dataset," 2020.

[50] R. Goyal, S. E. Kahou, V. Michalski, J. Materzyńska, S. Westphal, H. Kim, V. Haenel, I. Fruend, P. Yianilos, M. Mueller-Freitag, F. Hoppe, C. Thurau, I. Bax, and R. Memisevic, "The "something something" video database for learning and evaluating visual common sense," 2017.

[51] K. K. Reddy and M. Shah, "Recognizing 50 human action categories of web videos," Machine vision and applications, vol. 24, no. 5, pp. 971-981, 2013.

[52] J. Carreira, E. Noland, A. Banki-Horvath, C. Hillier, and A. Zisserman, "A short note about kinetics-600," 2018.

[53] J. Carreira, E. Noland, C. Hillier, and A. Zisserman, "A short note on the kinetics-700 human action dataset," 2019.

[54] F. Mahdisoltani, G. Berger, W. Gharbieh, D. Fleet, and R. Memisevic, "On the effectiveness of task granularity for transfer learning," 2018.

[55] F. Caba Heilbron, V. Escorcia, B. Ghanem, and J. Carlos Niebles, "Activitynet: A large-scale video benchmark for human activity understanding," in Proceedings of the ieee conference on computer vision and pattern recognition, 2015, pp. 961-970.

[56] S. Abu-El-Haija, N. Kothari, J. Lee, P. Natsev, G. Toderici, B. Varadarajan, and S. Vijayanarasimhan, "Youtube-8m: A large-scale video classification benchmark," arXiv preprint arXiv:1609.08675, 2016.

[57] A. Diba, M. Fayyaz, V. Sharma, M. Paluri, J. Gall, R. Stiefelhagen, and L. Van Gool, "Large scale holistic video understanding," in European Conference on Computer Vision. Springer, 2020, pp. 593-610.

[58] H. Zhao, A. Torralba, L. Torresani, and Z. Yan, "Hacs: Human action clips and segments dataset for recognition and temporal localization," 2019.

[59] J. Yue-Hei Ng, M. Hausknecht, S. Vijayanarasimhan, O. Vinyals, R. Monga, and G. Toderici, "Beyond short snippets: Deep networks for video classification," in Proceedings of the IEEE conference on computer vision and pattern recognition, 2015, pp. 4694-4702.

[60] Y. Zhu, X. Li, C. Liu, M. Zolfaghari, Y. Xiong, C. Wu, Z. Zhang, J. Tighe, R. Manmatha, and M. Li, "A comprehensive study of deep video action recognition," arXiv preprint arXiv:2012.06567, 2020.

[61] M. S. Ryoo, A. Piergiovanni, M. Tan, and A. Angelova, "Assemblenet: Searching for multi-stream neural connectivity in video architectures," in International Conference on Learning Representations, 2020. [Online]. Available: https://openreview.net/forum?id=SJgMK64Ywr

[62] X. Li, Y. Zhang, C. Liu, B. Shuai, Y. Zhu, B. Brattoli, H. Chen, I. Marsic, and J. Tighe, "Vidtr: Video transformer without convolutions," 2021.

[63] O. Russakovsky, J. Deng, H. Su, J. Krause, S. Satheesh, S. Ma, Z. Huang, A. Karpathy, A. Khosla, M. Bernstein et al., "Imagenet large scale visual recognition challenge," International journal of computer vision, vol. 115, no. 3, pp. 211-252, 2015.

[64] C. Sun, A. Shrivastava, S. Singh, and A. Gupta, "Revisiting unreasonable effectiveness of data in deep learning era," in Proceedings of the IEEE international conference on computer vision, 2017, pp. 843-852. 